\section{Vector for Positive Selection of In-Frame Genetic Sequences}

BioTechniques 23:619-621 (October 1997)

Polymerase chain reaction (PCR)based approaches are becoming increasingly important for the identification of members of extended multigene families as well as homologous gene structures present in phylogenetically divergent species $(2,5,10,11)$. Many of these approaches rely on the use of highly degenerate primers and/or reduced priming stringencies that can generate a broad range of products, including significant numbers of amplification products that contain frameshift(s) and/or termination codon(s). Recently, we introduced the use of short, minimally degenerate primers complementing conserved structural motifs for PCR amplification of homologs of antigenbinding receptor genes in phylogenetically diverse species $(6,8-10)$. This approach is also associated with the generation of amplification artifacts that require DNA sequencing to be distinguished from products that warrant further study. To facilitate identification of amplification products containing open reading frames, we have engineered a vector, pGFPfs, that affords positive selection of recombinants based on the continuity of coding sequence within a lacZ:insert:GFP (green fluorescent protein) fusion construct that is expressed in E. coli.

pGFPfs was derived from $\mathrm{pGFPuv}{ }^{\mathrm{TM}}$ (CLONTECH Laboratories, Palo Alto, CA, USA) by ligating a linker (formed by annealing the partially complementary oligonucleotides: 5'-GATCGATATCTCGAGT-3' and 5'-CTAGACTCGAGATATCGATCTGCA-3') into the multiple cloning site (MCS) of PstI/ $X b a$ I-digested pGFPuv. Incorporation of this linker, which was confirmed by DNA sequencing, disrupts the GFPuv reading frame and introduces additional unique restriction sites (Figure 1A). The GFP variant in these vectors, GFPuv, differs from wild-type GFP (7) in that it is optimized for bacterial expression, solubility and fluorescence of the isopropyl- $\beta$-D-thiogalactopyranoside (IPTG)-inducible lacZ:GFPuv fusion protein (3). Colonies producing GFPuv fusion proteins are identified by viewing under longwave UV light. Positive selection of recombinants containing in-frame inserts is based on correction of a frameshift that has been engineered within the $5^{\prime}$ MCS of pGFPfs.

PCR products were generated using methods that have been described previously by our laboratory (8-10) and were ligated into HindIII/XbaI-digested pGFPfs. Ligation mixtures were used to transfect $E$. coli $\mathrm{DH} 5 \alpha \mathrm{F}^{\prime \mathrm{TM}}$ (Life Technologies, Gaithersburg, MD, USA). Cells were plated on LB plates containing $10 \mathrm{mM}$ IPTG and $100 \mu \mathrm{g} / \mathrm{mL}$ ampicillin, and colonies were grown at $30^{\circ} \mathrm{C}$ for $48 \mathrm{~h}$.

Plasmid DNA was isolated for sequencing using a QIAprep ${ }^{\circledR}$ Spin Miniprep Kit (Qiagen, Chatsworth, CA, USA) in accordance with the manufacturer's recommended protocol. Sequencing was performed with a 4000L Automated Sequencer (LI-COR, Lincoln, NE, USA) using a SequiTherm ${ }^{\mathrm{TM}}$ LongRead $^{\mathrm{TM}}$ Cycle Sequencing Kit (Epicentre Technologies, Madison, WI, USA). Plates were viewed using a UVL 56 Blak-Ray ${ }^{\circledR}(366 \mathrm{~nm})$ longwave ultraviolet lamp (UVP, Upland, CA, USA).

IPTG induction of pGFPfs generates a 17-amino acid protein that is encoded by a fragment of lac $Z$, the contiguous cloning sites and a $3^{\prime}$ termination codon (Figure 1A); GFPuv is not expressed. Because cloning sites are $5^{\prime}$ of the GFPuv coding sequence, expression of GFPuv requires frame continuity within an insert. Therefore, directional cloning of PCR-derived products that are devoid of stop codons can be used to correct the reading frame to that of the GFPuv coding sequence (Figure 1B). Primers incorporating restriction sites can be designed to either remove the frameshift (e.g., HindIII/KpnI cloning) or introduce a second frameshift within the $3^{\prime}$ primer (e.g., HindIII/PstI cloning) to reestablish the correct reading frame and allow expression of GFPuv. The capacity of pGFPfs to discriminate between coding and noncoding amplification products is dependent on noncoding products having an internal stop codon(s) or shifted reading frame; i.e., \pm 1 base. It is apparent that $66 \%$ of noncoding products will not have the 
Table 1. pGFPfs Cloning

\begin{tabular}{|llc|}
\hline Sequencing Events* & green fluorescent colonies & 37 \\
Desirable Products & in-frame (ca. 200 bp) & 31 \\
Undesirable Products & in-frame artifacts (45-129 bp) & 5 \\
& out-of-frame (stop codon) & 1 \\
Efficiency & $(31 / 37)$ 84\% & \\
*As controls, 15 GFPuv-nonexpressing (white) colonies were recovered and \\
determined to be out-of-frame artifacts or vector.
\end{tabular}

correct number of nucleotides needed to restore the GFP coding sequence. However, the variable prevalence of stop codons in PCR-derived products provides an additional basis for exclusion of noncoding recombinants.

Validation studies of the pGFPfs construct were carried out initially by (re)amplification of previously characterized in-frame and out-of-frame

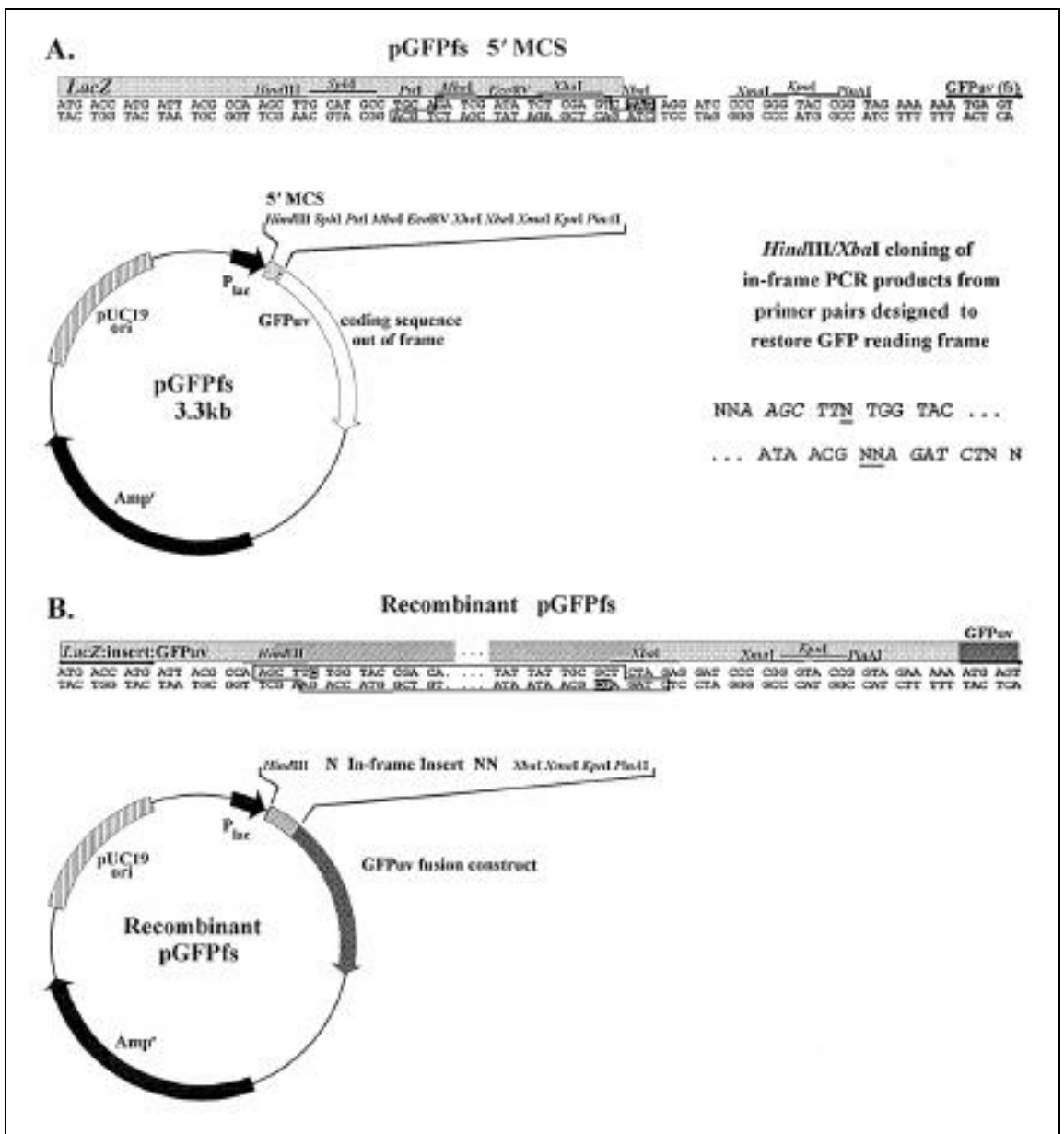

Figure 1. (A) pGFPfs vector diagram and $5^{\prime}$ MCS. The vector sequence shows the oligonucleotide linker (boxed), termination codon (reverse image) and the out-of-frame GFPuv coding sequence as well as restriction enzyme sites (labeled directly above the corresponding nucleotides). Other than the addition of the oligonucleotide linker, the vector is identical to pGFPuv. The 17-amino acid pGFPfs translation product is also shown above its coding sequence (stippled box). The circular vector diagram shows the out-of-frame GFPuv sequence (white). Shown to the right of the circular vector diagram are partial sequences of primer pairs used to correct the GFPuv reading frame; note the incorporation of nucleotides $(\underline{\mathrm{N}})$ used to maintain $\left(5^{\prime}\right)$ and correct $\left(3^{\prime}\right)$ product frame. (B) The lacZ:insert:GFPuv fusion protein is shown above its coding sequence; various stippling is used to indicate contributions from lac $Z$, insert and GFPuv and corresponds to the circular vector diagram. The nucleotides (N) that are introduced in this specific example to maintain continuous frame are shown (reverse text) within the insert-derived sequence (boxed). single-strand M13 templates, derived in the course of earlier studies (10). Sequencing of templates recovered from isolated nonfluorescent colonies transfected with pGFPfs or recombinant pGFPfs containing out-of-frame inserts as well as from fluorescent colonies transfected with recombinant pGFPfscontaining inserts with continuous reading frames confirmed the correlation $(100 \%)$ of fluorescence with in-frame sequences. Cloning of products derived by amplification with short, minimally degenerate primers using total mRNAderived cDNA as template yields GFPexpressing recombinants, nonexpressing recombinants and uncut vector. False-positive colonies have been observed and typically are the result of the integration of short inserts lacking termination codons that could not be eliminated by size selection. The generation of such interfering amplifications correlates directly with decreased primer stringency and/or template heterogeneity. In experiments using total mRNAderived cDNA as template, undesired in-frame recombinants represent less than $15 \%$ of positives. In our experience with conventional M13 cloning and sequencing of short primer amplification products (in excess of 1000 analyses), less than $15 \%$ of recombinants represent in-frame sequences (8-10). The use of pGFPfs cloning from total mRNA-derived cDNA resulted in a 5-6-fold overall improvement in the efficiency of identification of both known and novel in-frame sequences (Table 1). In-frame inserts of $>800 \mathrm{bp}$ have been shown to produce detectable GFPuv fusion proteins.

The vector can be utilized in searches for previously unrecognized members of extended multigene families. In addition, pGFPfs cloning can be used to identify out-of-frame sequences, such as those arising from diseasecausing mutations. For such an application, which could serve as a primary diagnostic tool, amplifications would be carried out under highly stringent conditions using primers that are designed to require a frameshift (associated with disease) immediately upstream of the $3^{\prime}$ primer to rescue GFP expression; amplification of the corresponding normal sequence would not rescue GFP expression. In a general sense, pGFPfs is 
a convenient, highly efficient tool for distinguishing in-frame from out-offrame and/or termination codon-containing sequences and has potential application for distinguishing wild-type and certain mutant sequences.

\section{REFERENCES}

1.Barclay, A.N., M.L. Birkeland, M.H. Brown, A.D. Beyers, S.J. Davis, C. Somoza and A.F. Williams. 1993. The Leucocyte Antigen FactsBook. Academic Press, San Diego.

2.Bozdech, Z., U. Delling, S.K. Volkman, A.F. Cowman and E. Schurr. 1996. Cloning and sequence analysis of a novel member of the ATP-binding cassette (ABC) protein gene family from Plasmodium falciparum. Mol. Biochem. Parasitol. 81:41-51.

3.Crameri, A., E.A. Whitehorn, E. Tate and W.P.C. Stemmer. 1996. Improved green fluorescent protein by molecular evolution using DNA shuffling. Nat. Biotechnol. 14:315-319.

4.Kabat, E.A., T.T. Wu, C. Foeller, H.M. Perry and K. Gottesman. 1991. Sequences of Proteins of Immunological Interest. U.S. Dept. Health and Human Services, Washington, D.C.
5.Kim, Y.W., H. Wang, I. Sures, R. Lammers, K.J. Martell and A. Ullrich. 1996. Characterization of the PEST family protein tyrosine phosphatase BDP1. Oncogene 13:2275-2279.

6.Partula, S., A. de Guerra, J.S. Fellah and J. Charlemagne. 1995. Structure and diversity of the $T$ cell antigen receptor $\beta$-chain in a teleost fish. J. Immunol. 155:699-706.

7.Prasher, D.C., V.K. Eckenrode, W.W. Ward, F.G. Prendergast and M.J. Cormier. 1992. Primary structure of the Aequorea victoria green-fluorescent protein. Gene 111:229-233.

8.Rast, J.P., M.K. Anderson, S.J. Strong, C. Luer, R.T. Litman and G.W. Litman. 1997. $\alpha, \beta, \gamma$, and $\delta \mathrm{T}$ cell antigen receptor genes arose early in vertebrate phylogeny. Immunity 5:1-11.

9.Rast, J.P., R.N. Haire, R.T. Litman, S. Pross and G.W. Litman. 1995. Identification and characterization of T-cell antigen receptor related genes in phylogenetically diverse vertebrate species. Immunogenetics 42:204-212.

10.Rast, J.P. and G.W. Litman. 1994. T cell receptor gene homologs are present in the most primitive jawed vertebrates. Proc. Natl. Acad. Sci. USA 91:9248-9252.

11.Yoshihara, Y., M. Kawasaki, A. Tani, A. Tamada, S. Nagata, H. Kagamiyama and K. Mori. 1994. BIG-1: a new TAG-1/F3-relat- ed member of the immunoglobulin superfamily with neurite outgrowth-promoting activity. Neuron 13:415-426.

This work was supported by grants to G.W.L. from the National Institutes of Health (Nos. R03 AI40137 and R37 AI23338). Address correspondence to Gary W. Litman, Department of Pediatrics, University of South Florida, All Children's Hospital, 801 Sixth Street South, St. Petersburg, FL 33701, USA. Internet: litmang@allkids.org

Received 27 February 1997; accepted 30 April 1997.

Noel A. Hawke, Scott J. Strong, Robert N. Haire and Gary W. Litman University of South Florida Tampa All Children's Hospital St. Petersburg, FL, USA 